\title{
Representaciones sociales de estudiantes universitarios sobre el papel de la universidad en la creación de cultura de paz ${ }^{1}$
}

\author{
Jesús Ernesto Urbina Cárdenas ${ }^{2}$ \\ Gustavo Adolfo Ovalles Rodríguez ${ }^{3}$ \\ Blanca Johanna Pérez Fernández ${ }^{4}$
}

Recibido: 16-08-2017

Aceptado: 13-10-2017

\section{RESUMEN}

El artículo muestra los resultados de la investigación sobre el tipo de representaciones sociales que poseen los estudiantes de la UFPS, acerca del papel de la universidad en la creación de una cultura de paz. Las categorías centrales sobre las cuales se fundamenta son las siguientes: la teoría de las representaciones sociales (RS) ${ }^{5}$ como marco teóricoepistemológico para la interpretación de los fenómenos socio-educativos y el concepto de Cultura de Paz. El texto expone la parte descriptiva del

1. Este artículo se origina en la investigación titulada “ ¿̇Y si no me reconozco en él? , ¿quién soy? Representaciones sociales de estudiantes universitarios sobre el papel de la universidad en la creación de una cultura de paz", financiado por el Fondo de Investigaciones de la Universidad Francisco de Paula Santander (FINU) código 043. 2015.

2. Licenciado en Filosofía-letras con título de Doctor en Ciencias Sociales, Posdoctor en Educación Intercultural y Magíster en pedagogía. Profesor titular tiempo completo adscrito al Departamento de Ciencias Humanas, Sociales e Idiomas de la Universidad Francisco de Paula Santander, y Director del Grupo de Investigación en Estudios Sociales y Pedagogía para la Paz -Giesppaz-, categoría A, Colciencias. ORCID: 0000-0002-5262-9527

3. Ingeniero químico con título de Magíster en Práctica Pedagógica, Especialista en Estadística Aplicada. Profesor catedrático adscrito al Departamento de Matemáticas y Estadística, tutor de la línea de investigación en Pedagogía para la Paz de la Maestría en Práctica pedagógica de la Universidad Francisco de Paula Santander de Cúcuta. ORCID: 0000-0001-7196-1560

4. Profesional en Salud Ocupacional con maestría en Administración de Empresas con énfasis en Sistemas Integrados de Gestión, Especialización en Gerencia Social, adscrita al Departamento de Procesos Industriales de la Universidad Francisco de Paula Santander y Directora del Semillero de Investigación en Pedagogía para la Prevención de Riesgos Laborales - Sipprila UFPS.

ORCID: 0000-0003-2366-0952

5. RS: Representación Social. 
estudio, a partir de una encuesta aplicada a quinientos (500) estudiantes de las distintas carreras de la universidad. Algunos de los resultados permiten percibir cierto escepticismo frente al proceso de paz y una falta de conocimiento sobre los avances y logros de los diálogos de La Habana. El 59.9\% afirma no conocer lo que se está pactando en la isla, el $37.4 \%$ cree que nunca se va a firmar la paz, el conflicto armado se asocia con la inequidad social en un $54 \%$ de las respuestas, la palabra con la que asocian la paz, en mayor porcentaje, es "la justicia" en un 34.8\%, el 45.3\% considera que la universidad debe abrir debates permanentes como principal estrategia para construir cultura de paz, y el $57.7 \%$ afirma que no hay un buen ambiente en la universidad para construir dicha cultura.

Palabras clave: paz, cultura de paz, representaciones sociales, conflicto.

\title{
Social representations of university students on the role of the university in creating a culture of peace
}

\begin{abstract}
This article shows the results of research on the type of social representations that have students of Universidad Francisco de Paula Santander (UFPS) about the role of universities in creating a culture of peace. The central categories on which it is based are as follows: the theory of social representations (RS) as epistemological theoretical framework for the interpretation of the socio-educational phenomena and the concept of Culture of Peace. The paper exposes the descriptive part of the study from a survey, this survey was applied to 500 students from different academic programs of the University. Some of the results of sensing a certain skepticism about the peace process and a lack of knowledge about the progress and achievements of the dialogues in Havana. $59.9 \%$ of respondents said that they don't know what is agreeing on the island, $37.4 \%$ believe it will never make peace, armed conflict is associated with social inequity by $54 \%$ of respondents, the word that peace associated higher percentage is "justice" in 34.8\%, 45.3\% believe that the university should open ongoing discussions as the main strategy to build a culture of peace and $57.7 \%$ said that there is a good atmosphere in the university to build that culture.
\end{abstract}


Keywords: peace, peace culture, social representations, conflict.

\section{Introduccion}

La situación de Cúcuta, capital del departamento Norte de Santander, ubicada en la frontera con la República Bolivariana de Venezuela, es compleja. Según datos del Departamento Administrativo Nacional de Estadística - DANE (febrero de 2016), la capital de la frontera es la segunda ciudad de Colombia con mayor desempleo (15.3\%), después de Quibdó con el 16.1\%. En el 2015, Cúcuta estaba entre las 50 ciudades más violentas del mundo, y la región del Catatumbo (en el mismo departamento) tiene 6.345 hectáreas sembradas con cultivos ilícitos (el $13.16 \%$ del país).

Esta situación crítica de la Frontera, y en particular las consecuencias que este fenómeno produce en el ámbito regional, motiva un conjunto de interrogantes sobre el papel que debe jugar la universidad en estos asuntos. Ahora bien, esta es un sistema abierto y flexible en el que convergen múltiples cosmovisiones, y cuya misión de docencia, investigación y extensión social, configuran un universo de ideas, ideologías y posiciones en los ámbitos filosóficos, científicos, sociales, políticos y culturales. Precisamente, es en las instituciones de educación superior, donde la sociedad deposita todas sus esperanzas de progreso y desarrollo social (Urbina, 2012).

Sin embargo, no siempre estos propósitos de orden estructural se cumplen. La universidad, queda reducida a una fábrica de profesionales, muchas veces sin guardar correspondencia con los requerimientos del entorno, y proyectando su accionar de espaldas a los grandes interrogantes que formula el contexto. En la práctica ocurre un divorcio entre la academia y las demandas de una sociedad, ensimismada en sus propios desafíos y enfrentada a los dilemas de su tiempo (Urbina, 2012).

Estos asuntos críticos llevaron al Grupo de Investigación en Estudios Sociales y Pedagogía para la Paz - Giesppaz- de la Universidad Francisco de Paula Santander de Cúcuta a plantearse una serie de preguntas: ¿Cuál es el papel de la universidad pública regional frente a la consecuencias del conflicto armado en la frontera colombo-venezolana y cómo afrontar un posible escenario de posconflicto? ¿Este papel se limita a la 
implementación de una cátedra de la paz? ¿El asunto se resuelve creando una asignatura como problema en el currículo?

Preguntas que al interior del Grupo de Investigación Giesppaz, necesariamente conducen a un replanteamiento de las funciones de la universidad, y le otorga relevancia a uno de los principios orientadores del grupo: un actuar reflexivo motivado por una actitud y una vocación por la emancipación. Entendida la emancipación en el sentido expuesto por Habermas (1984), como un proceso de cambio e innovación permanente de los sujetos comprometidos en la construcción de una sociedad más equitativa. Por esta razón, las preguntas desencadenantes de la presente investigación poseen una orientación en esta perspectiva: ¿Qué acciones realizar para consolidar desde la academia una cultura de paz? ¿Cómo transformar los currículos universitarios desde el punto de vista de una pedagogía para la paz? ¿Qué piensan y qué sienten los miembros de la comunidad universitaria en torno al conflicto, la violencia, la guerra, la paz y el posconflicto? Y de ser necesario ¿Qué acciones pedagógicas emprender para construir una nueva mirada y una nueva cultura sobre estos fenómenos?

Para dar respuesta a algunos de estos interrogantes, se explora el problema desde la perspectiva de la teoría de las representaciones sociales. La teoría clásica de las representaciones sociales tiene su origen en Moscovici (1979), Denise Jodelet (1984), Willem Doise (1991), Jean-Claude Abric (1994). Se asumen las representaciones sociales como la manera en que los sujetos sociales aprehenden los hechos cotidianos, las características del entorno, y las informaciones que en él circulan, en tópicos relacionados con la paz, el conflicto y el posconflicto. También se definen como los conocimientos específicos y los saberes del sentido común que orientan nuestras acciones, la comunicación y la comprensión del entorno social, ideal o material sobre este tema.

El origen de esta teoría se remonta al año de 1961, cuando Serge Moscovici presenta su tesis doctoral titulada "La Psychoanalyse, son image et son public", e introduce el mencionado concepto. El autor analizó las formas como la sociedad francesa representaba el psicoanálisis, utilizando como fuentes de información la prensa y la entrevista en profundidad a diversos grupos sociales. Al definir las representaciones sociales como "explicaciones del sentido común", sienta las bases sobre la comprensión de los fenómenos sociales de una manera más dinámica y fluida, en comparación con la propuesta 
de Durkheim y su idea de las "representaciones colectivas" (Durkheim, 1898).

Moscovici (1979), propone tres tipos de representaciones sociales: las representaciones hegemónicas, relacionadas con el consenso entre miembros de un grupo social; las representaciones emancipadas, que emergen entre sub-grupos específicos con nuevas formas de pensamiento social; y finalmente, las representaciones polémicas, cuando se presentan controversia y son portadoras de nuevas formas de pensamiento divergente.

Una de las continuadoras de esta corriente de pensamiento social es Denis Jodelet (1984, 1989); quien sostiene que el concepto de representación social se refiere "al saber del sentido común", y cuyas principales elaboraciones se plantean, según Perera (2003, p. 10), en los siguientes aspectos:

- La manera en que nosotros, sujetos sociales aprendemos los acontecimientos de la vida diaria, las características de nuestro medio ambiente, las informaciones que en él circulan, a las personas de nuestro entorno próximo o lejano.

- El conocimiento espontáneo, ingenuo o de sentido común, por oposición al pensamiento científico.

- Conocimiento socialmente elaborado y compartido, constituido a partir de nuestras experiencias y de las informaciones y modelos de pensamiento que recibimos y transmitimos a través de la tradición, la educación y la comunicación social.

- Conocimiento práctico que participa en la construcción social de una realidad común a un conjunto social e intenta dominar esencialmente ese entorno, comprender y explicar los hechos e ideas de nuestro universo de vida.

- Son a un mismo tiempo producto y proceso de una actividad de apropiación de una realidad externa y de elaboración psicológica y social de esa realidad. Son pensamiento constitutivo y constituyente.

Willem Doise (1991), orienta su trabajo hacia la comprensión de las condiciones en que se producen las representaciones sociales, y se inclina por el método estadístico correlacional. Jean-Claude Abric, (1994) propone que toda representación se organiza en torno a un núcleo 
central y un núcleo periférico, de tal modo "los elementos cognitivos del núcleo se caracterizan por mayor estabilidad, rigidez y consensualidad; en tanto el sistema periférico adopta mayor dinamismo, flexibilidad e individualización. La representación se estructura en torno al núcleo, que la dota de significación global y organiza los elementos periféricos, que gracias a su mutabilidad permiten una relativa armonía en las situaciones y prácticas concretas de la cotidianidad" (Perera, 2003, p. 14).

La cultura de paz (Tuvilla, 2006; Jares, 1999; Lederach, 2000, 2008; Fisas, 1998 y Vicent Martínez, 2000), se refiere al conjunto de valores, actitudes, tradiciones, comportamientos y estilos de vida que propician una manera distinta de concebir el mundo y de vivir juntos, con el fin de aprender a resolver de manera creativa y pacífica los conflictos que surjan de esa convivencia en común. Para estos autores, la paz es real y se manifiesta como un fenómeno visible y palpable para los seres humanos: así como es posible evidenciar "hechos violentos", también existen "hechos de paz".

Jares (1999), cita un grupo de investigadores clásicos que permiten realizar un seguimiento al concepto tradicional de paz: Galtung (1985 y 2003); Lederach (2000 y 2008) Curle (1978). En conclusión, para estos filósofos y pedagogos para la paz es preciso diferenciar entre el concepto tradicional de "Paz negativa" y el concepto de "Paz positiva". La paz negativa se relaciona con la idea de ausencia total de conflicto, situación que iría en contravía de un rasgo connatural al ser humano y su carácter conflictual. Esta visión supone que habrá paz cuando no haya guerra.

La paz positiva, por el contrario, no se relaciona solamente con ausencia de guerra, sino con la ausencia de violencia estructural y cultural (Galtung, 1969). Es decir, comprende un concepto mucho más amplio y complejo, en donde la paz no es un estado ideal donde se debe eliminar el conflicto, sino una búsqueda permanente (paz imperfecta, Muñoz, 2001, 2010) para lograr la armonía social, la justicia y la igualdad: la paz no es un fin, es un proceso (Mesa, 2009). La Tabla No 1, tomada del artículo "paz y seguridad" de Manuela Mesa Peinado (2009), diferencia estos dos conceptos: 


\begin{tabular}{|c|c|c|}
\hline & Paz Negativa & Paz positiva \\
\hline Meta & -Evitar los conflictos armados. & $\begin{array}{l}\text {-Lograr la armonía social, } \\
\text { justicia e igualdad y la } \\
\text { eliminación de la violencia } \\
\text { estructural. }\end{array}$ \\
\hline Características & $\begin{array}{l}\text {-Ausencia de guerra o } \\
\text { violencia directa. } \\
\text { - Se establece entre Estados } \\
\text { y requiere de un aparato } \\
\text { militar que garantice la paz. }\end{array}$ & $\begin{array}{l}\text {-Ausencia de violencia } \\
\text { directa, estructural y cultural. } \\
\text {-Reducido nivel de violencia } \\
\text { directa y elevado nivel de } \\
\text { justicia. }\end{array}$ \\
\hline Estrategias & $\begin{array}{l}\text { Se admite la "guerra pacífica" } \\
\text { por medio de sanciones } \\
\text { económicas, boicots } \\
\text { comerciales, etc. }\end{array}$ & $\begin{array}{l}\text { La paz no es un fin, es un } \\
\text { proceso. No supone un } \\
\text { rechazo del conflicto, al } \\
\text { contrario. Los conflictos hay } \\
\text { que aprender a afrontarlos } \\
\text { y resolverlos de manera } \\
\text { pacífica y justa. }\end{array}$ \\
\hline
\end{tabular}

Tabla 1. Fuente: Manuela Mesa (2009).

Para otros autores (citados por Jares, 1999) como Betty Reardom (1978), la paz constituye el conjunto de las relaciones humanas, en el cual la justicia social y la equidad pueden lograrse sin hechos violentos. Naidu (1986) propone cinco dimensiones para entender la paz: no violencia, justicia económica, igualdad social, libertad política y fraternidad psicológica.

Para la Unesco (1974), la paz no es sólo ausencia de conflicto armado, también implica un proceso en el que el progreso, la justicia y el respeto mutuo entre los pueblos se constituyen en pilares para la convivencia social. Por esa razón, el trabajo de Johan Galtung (1969, 1985), citado por Manuela Mesa (2009), sobre violencias invisibles y violencias visibles es fundamental:

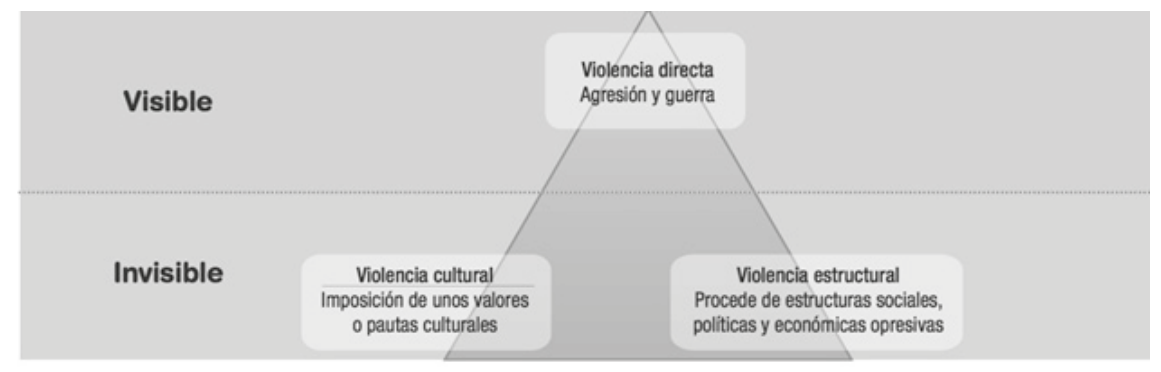

Figura 1. Tipos de violencia. Fuente: Manuela Mesa (2009). 
A partir de estos supuestos teóricos, y situados desde el aporte robusto de la Teoría de las Representaciones Sociales, el artículo muestras algunas de las representaciones sobre cultura de paz y universidad, y otros conceptos adyacentes al problema de investigación, analizadas desde los resultados de una encuesta.

\section{Metodología}

Aunque la investigación se desarrolló con una clara intención de comprender las representaciones sociales sobre el papel de la universidad en la construcción de una cultura de paz, se utilizó inicialmente una encuesta cerrada de corte cuantitativo conformada por 20 preguntas aplicada a 500 estudiantes de distintas carreras de la UFPS, con el fin de configurar de forma preliminar las categorías. Tomando como base este ejercicio, se seleccionaron posteriormente 25 participantes para el desarrollo de igual número de entrevistas en profundidad. Este artículo da cuenta solamente de los resultados de la encuesta.

La información proveniente de las encuestas fue tabulada y clasificada con ayuda del software estadístico R. Cada una de las preguntas de la encuesta estaba conformada por cierto número de categorías cualitativas que correspondían a las diferentes opciones de respuesta. Para cada pregunta se realizaron análisis estadísticos descriptivos que permitieron obtener cuadros de salida con las frecuencias porcentuales de las categorías, así como su representación en gráficos de barras. Este ordenamiento y clasificación de la información permitió el análisis de los resultados.

\begin{tabular}{|l|l|l|}
\hline \multicolumn{1}{|c|}{ Programa académico } & Hombres & Mujeres \\
\hline Administración de Empresas & 21 & 21 \\
\hline Comercio Internacional & 15 & 15 \\
\hline Ingeniería de Sistemas & 15 & 15 \\
\hline Ingeniería Mecánica & 17 & 07 \\
\hline Ingeniería de Minas & 16 & 08 \\
\hline Ingeniería Electrónica & 18 & 06 \\
\hline Ingeniería Industrial & 16 & 16 \\
\hline Enfermería & 04 & 20 \\
\hline Trabajo Social & 15 & 65 \\
\hline Comunicación Social & 10 & 30 \\
\hline
\end{tabular}




\begin{tabular}{|l|l|l|}
\hline \multicolumn{1}{|c|}{ Programa académico } & Hombres & Mujeres \\
\hline Derecho & 20 & 20 \\
\hline Arquitectura & 10 & 10 \\
\hline Licenciatura en Matemáticas & 05 & 05 \\
\hline Ingeniería Biotecnológica & 08 & 08 \\
\hline Ingeniería Ambiental & 12 & 12 \\
\hline Tecn. Regencia en Farmacia (Distancia) & 07 & 13 \\
\hline Tecn. Administración Financiera (Dist.) & 08 & 12 \\
\hline TOTAL & 217 & 283 \\
\hline
\end{tabular}

Tabla 2. Distribución de estudiantes por carrera y por género que respondieron la encuesta.

\section{Objetivos}

\section{Objetivo general}

Comprender las representaciones sociales de un grupo de estudiantes universitarios sobre el papel de la universidad en la creación de una cultura de paz.

\section{Objetivos específicos}

- Realizar una aproximación a la Teoría de las Representaciones Sociales a partir del análisis de una encuesta orientada al estudio del conocimiento cotidiano de estudiantes universitarios sobre el papel de la universidad en la creación de una cultura de paz.

- Identificar las representaciones que poseen los estudiantes universitarios sobre paz, cultura de paz, escenarios de paz, conflicto y posconflicto.

\section{Resultados y discusión}

\section{La representación de la realidad del país}

A continuación, se muestran algunas de las representaciones sociales (RS) de los estudiantes universitarios (EU), sobre diferentes tópicos 
relacionados con el conflicto armado que sufre el país, la paz, la guerra, las políticas prioritarias del Estado, y el papel de la universidad en este proceso. Es importante destacar al inicio de la presentación de estos resultados, que el $32.4 \%$ de la muestra total de estudiantes (500), manifiestan haber sido víctimas del conflicto armado, de los cuales el $46 \%$ fueron afectados por el asesinato de un familiar, el $26.7 \%$ desplazamiento forzado, el $12.7 \%$ amenaza de muerte, el 7.3\% secuestro y extorsión respectivamente cada una.

En síntesis, tres de cada diez estudiantes de la muestra, han sido víctimas del conflicto armado en Colombia. Con esta población se indagó en las RS sobre conflicto y paz.

Los estudiantes universitarios representan la realidad del país con palabras como "inseguridad", "conflicto" y "atraso":

\begin{tabular}{|l|l|}
\hline \multicolumn{1}{|c|}{$\begin{array}{c}\text { Palabras que mejor representan la } \\
\text { realidad del pais }\end{array}$} & $\begin{array}{c}\text { Frecuencia } \\
\text { Porcentual }\end{array}$ \\
\hline Inseguridad & 27.7 \\
\hline Conflicto & 24.9 \\
\hline Atraso & 16.2 \\
\hline Optimismo & 10.7 \\
\hline Violencia & 8.5 \\
\hline Progreso & 5.5 \\
\hline Paz & 2.3 \\
\hline Pesimismo & 4.0 \\
\hline Tranquilidad & 0.2 \\
\hline
\end{tabular}

Tabla 3. Lista de palabras y porcentajes: así representan el país los EU. ${ }^{6}$

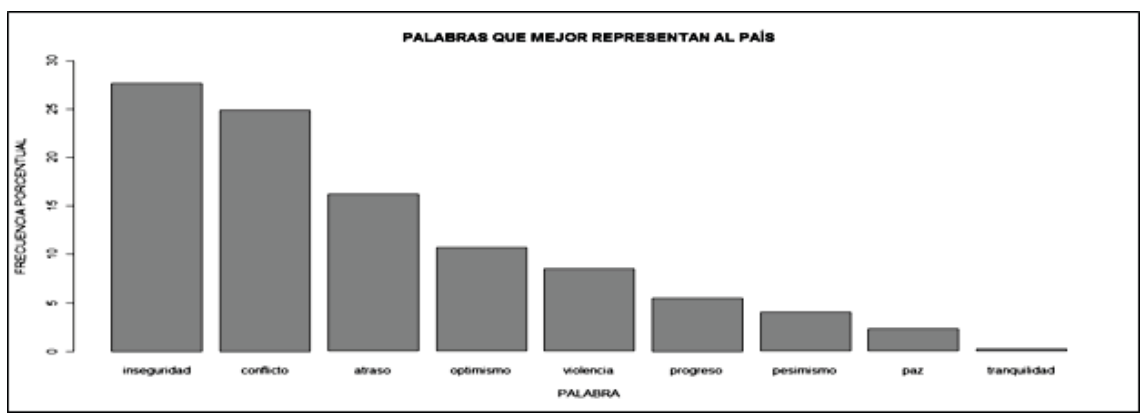

Gráfico 1. Palabras que representan la realidad del país para estudiantes universitarios. 
Esta representación de la realidad del país con la palabra "inseguridad" $(27.7 \%)$, corresponde con uno de los mayores flagelos sociales que afectan a los colombianos, y que los medios masivos de información exponen a diario en los noticieros. La Red de ciudades cómo vamos ${ }^{7}$, que anualmente mide las percepciones de los ciudadanos de 14 ciudades del país, concluye que sólo 3 de cada 10 habitantes del país se siente seguro. Por su parte, el Departamento Administrativo Nacional de Estadística (DANE), reporta en el 2015 las siguientes cifras para Cúcuta: el 13,7\% manifiesta haber sido víctima de algún delito, el $4.0 \%$ fue la tasa de hurto a residencias por encima de la media nacional que fue del $2.7 \%$, el hurto a personas fue del $6.2 \%$ del $10.5 \%$ de la media nacional, y el $49.4 \%$ manifiesta sentirse insegura en la ciudad.

La segunda representación se enfoca a identificar cuál es el mayor problema que tiene el país actualmente, y tiene como objetivo analizar desde la perspectiva de los estudiantes los fenómenos sociales, políticos y económicos que caracterizan a Colombia:

\begin{tabular}{|l|l|}
\hline \multicolumn{1}{|c|}{ Mayor problema que tiene el pais actualmente } & Frecuencia Porcentual \\
\hline Corrupción & 64.1 \\
\hline Conflicto armado & 11.6 \\
\hline Inequidad social & 10.9 \\
\hline Falta de oportunidades & 6.0 \\
\hline Narcotráfico & 2.9 \\
\hline Otra & 4.5 \\
\hline
\end{tabular}

Tabla 4. El mayor problema del país según los EU

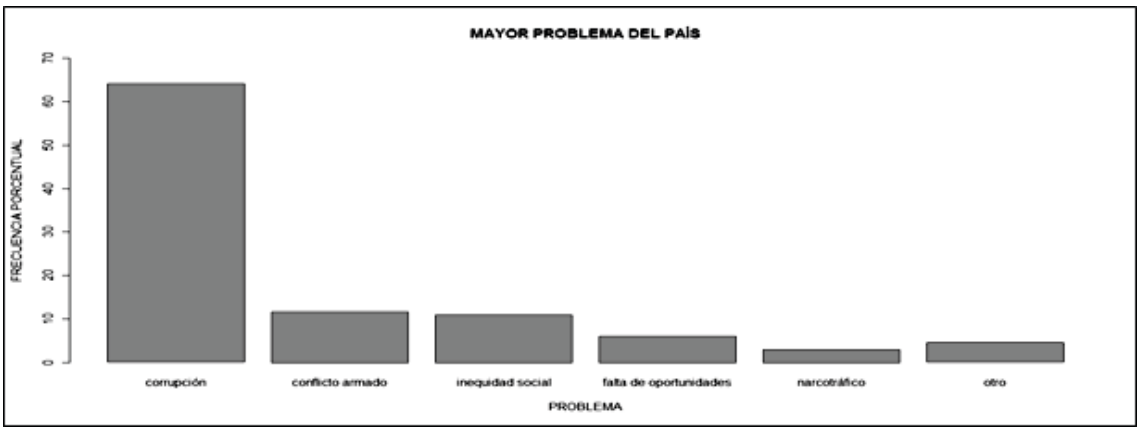

Gráfico 2. El mayor problema del país según la RS de los EU.

Según los estudiantes universitarios, el mayor problema que tiene el país es la corrupción con un $64.1 \%$, muy por encima de fenómenos como el conflicto armado y la inequidad social. Este dato se relaciona con 
la cifra publicada por Transparencia Internacional, según la cual, en el año 2015, Colombia obtuvo un puntaje de 37 sobre 100, entre los países más corruptos del mundo, ocupando el puesto 83 entre 168 países. Esta investigación, al menos de forma preliminar, muestra cómo nuestros jóvenes representan esta realidad nacional.

En cuanto a la política prioritaria para el país, los jóvenes la representan con una mayor inversión en educación (36.7\%), y, en segundo lugar, en más fuentes de empleo (24.8\%), tal como lo evidencia la tabla 5 :

\begin{tabular}{|l|l|}
\hline \multicolumn{1}{|c|}{ Política de Estado prioritaria } & Frecuencia porcentual \\
\hline Mayor inversión en educación & 36.7 \\
\hline Más fuentes de empleo & 24.8 \\
\hline Cese del conflicto armado & 17.8 \\
\hline Inversión en el campo & 7.2 \\
\hline Apoyo a las microempresas & 5.9 \\
\hline Fortalecimiento de programas sociales & 3.4 \\
\hline Otra & 4.2 \\
\hline
\end{tabular}

Tabla 5. . La política prioritaria para el país según la RS de EU.

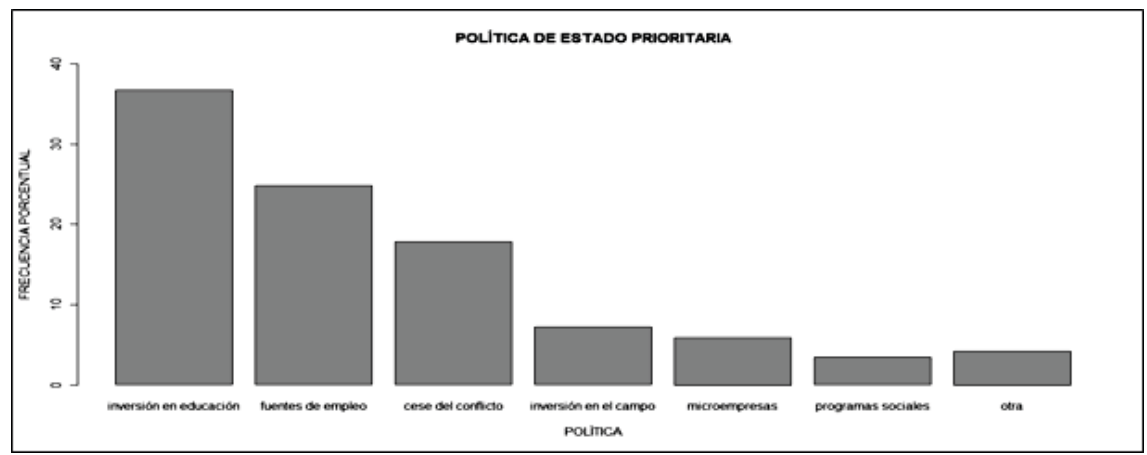

Gráfico 3. RS de los EU sobre las políticas prioritarias que debe asumir el gobierno.

En síntesis, para los EU la representación social del país se resume en tres palabras: inseguridad, corrupción y la necesidad de mayor educación. Estas maneras de representar la realidad nacional se aproximan a uno de los postulados señalados por Perera (2003), tomando a Jodelet: las RS son el conocimiento práctico que actúa en la construcción social de una realidad afín a un conjunto social y procura manipular ese contexto, comprender y explicar los fenómenos e ideas de nuestro mundo de la vida. 


\section{Representaciones sociales sobre conflicto armado}

Un dato importante es la aproximación de esta investigación a la manera como los universitarios representan la guerra y el conflicto. La guerra la asocian con impunidad (39.7\%) y desigualdad (20.8\%). Según el Índice Global de Impunidad (IGI, 2015), Colombia es el tercer país con mayor impunidad (75.6\%), después de Filipinas (80\%) y México (75.7\%). Datos que muestran la manera como nuestros jóvenes universitarios asocian el origen de la guerra en Colombia: mientras haya impunidad y desigualdad, habrá guerra en el país:

\begin{tabular}{|l|l|}
\hline $\begin{array}{c}\text { Palabra que mejor representa la guerra } \\
\text { en Colombia }\end{array}$ & $\begin{array}{c}\text { Frecuencia } \\
\text { porcentual }\end{array}$ \\
\hline Impunidad & 39.7 \\
\hline Desigualdad & 20.8 \\
\hline Muerte & 18.7 \\
\hline Horror & 10.4 \\
\hline Otra & 5.4 \\
\hline Supervivencia & 4.0 \\
\hline Defensa propia & 1.0 \\
\hline
\end{tabular}

Tabla 6. La guerra según los estudiantes universitarios.

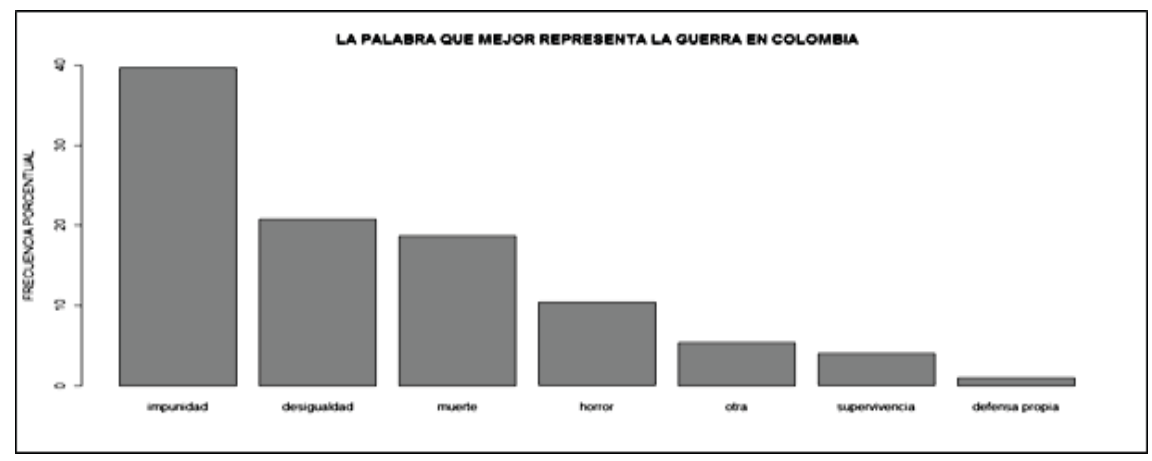

Gráfico 4. RS de la guerra en Colombia.

La investigación indagó en los EU sobre la representación del conflicto. Es importante señalar que "guerra" y "conflicto" no son palabras sinónimas. La guerra es una manera de manifestarse el conflicto, del mismo modo que la actuación pacífica, corresponde a otra expresión del conflicto (Urbina \& Muñoz, 2012). Para los EU el conflicto se relaciona 
con la "problemática social" en un $54.1 \%$ y la guerra está representada en un $17.3 \%$. En esta representación es importante destacar la manera como los jóvenes conciben el conflicto, no simplemente como la guerra abierta entre el Estado y los grupos insurgentes, sino como uno de los factores de mayor incidencia y una de las cusas históricas del conflicto armado en Colombia, además del tema asociado a la tierra y al agro (Fajardo, 2014).

\begin{tabular}{|l|l|}
\hline \multicolumn{1}{|c|}{$\begin{array}{c}\text { Palabra que mejor representa el } \\
\text { conflicto en Colombia }\end{array}$} & \multicolumn{1}{|c|}{$\begin{array}{c}\text { Frecuencia } \\
\text { porcentual }\end{array}$} \\
\hline Problemática social & 54.1 \\
\hline Guerra & 17.3 \\
\hline Punto de vista diferente & 12.6 \\
\hline Asesinato & 5.7 \\
\hline Otra & 5.3 \\
\hline Dificultad de comunicación & 5.0 \\
\hline
\end{tabular}

Tabla 7. El conflicto según los EU.

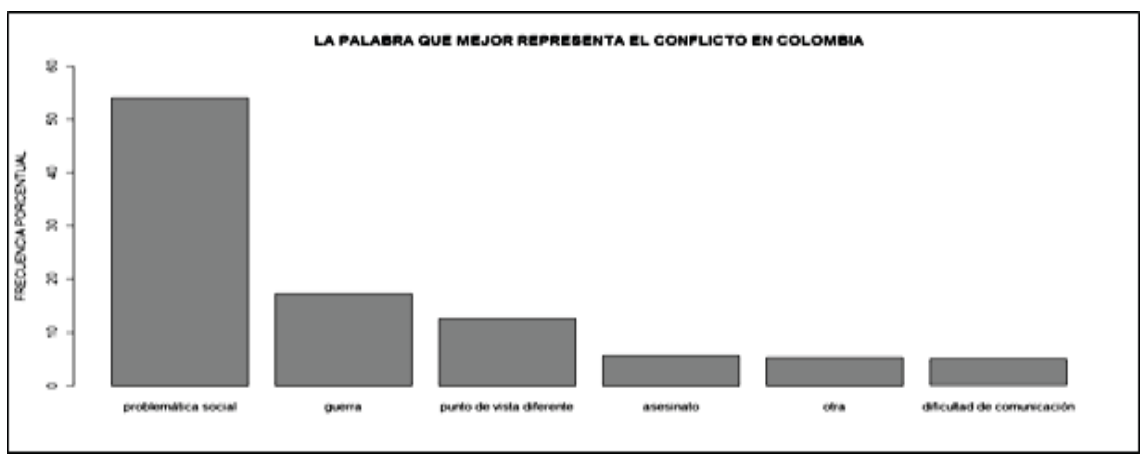

Gráfico 5. RS del conflicto según los estudiantes universitarios.

\section{RS sobre los diálogos de paz entre el Gobierno $y$ las Farc en la Habana}

La investigación arroja un dato preocupante sobre el desconocimiento de los EU sobre los acuerdos a que está llegando el Gobierno colombiano con las Farc en la Habana: el 59.9\% manifiesta desconocer el resultados de los diálogos en Cuba. A este dato, que puede generar inquietud, se suma, el pesimismo de los EU sobre el tiempo que puede durar la firma del cese al conflicto armado: para el 37.4\% la firma no se concretará nunca, mientras que el $21.5 \%$, considera que puede extenderse a más de un año. Estas representaciones se aproximan a las cifras publicadas por 
los medios sobre el pesimismo de los colombianos frente al proceso de paz y la economía (Semana, 2015; Colombia.com, 2016).

La siguiente tabla y el gráfico 6 muestra esta representación del pesimismo sobre los acuerdos de paz:

\begin{tabular}{|l|l|}
\hline \multicolumn{1}{|c|}{$\begin{array}{c}\text { Tiempo para la firma del acuerdo de } \\
\text { paz }\end{array}$} & \multicolumn{1}{c|}{$\begin{array}{c}\text { Frecuencia } \\
\text { porcentual }\end{array}$} \\
\hline Nunca & 37.4 \\
\hline Más de un año & 21.5 \\
\hline Antes de un año & 18.4 \\
\hline Tres años & 9.0 \\
\hline Dos años & 8.4 \\
\hline Otro & 5.3 \\
\hline
\end{tabular}

Tabla 8. La RS sobre el tiempo de duración de los acuerdos de paz.

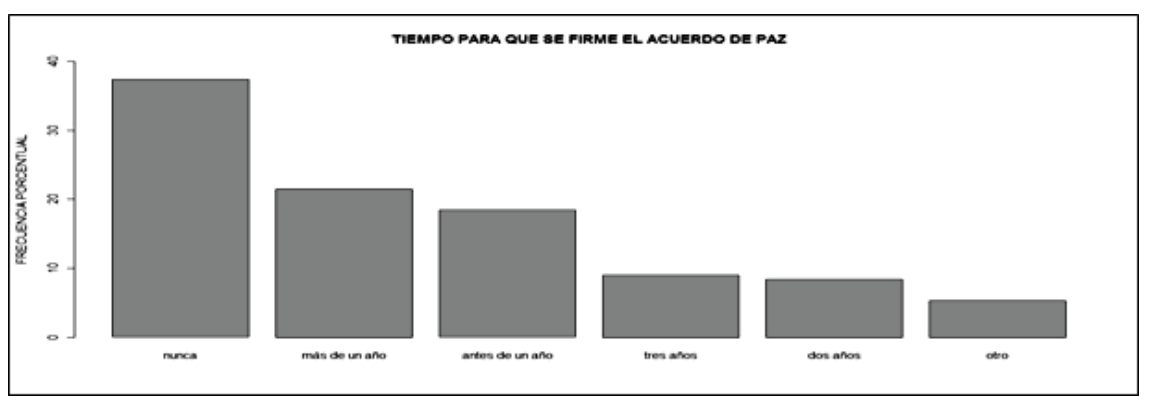

Gráfico 6. Tiempo para la firma del acuerdo de paz entre el gobierno y las Farc.

Los estudiantes universitarios representan de la siguiente manera los principales factores que impiden la paz en Colombia:

\begin{tabular}{|l|l|}
\hline \multicolumn{1}{|c|}{ Principal factor que impide la paz } & \multicolumn{1}{c|}{$\begin{array}{c}\text { Frecuencia } \\
\text { porcentual }\end{array}$} \\
\hline Narcotráfico & 21.3 \\
\hline Inseguridad & 19.6 \\
\hline Desempleo & 18.7 \\
\hline La guerrilla & 17.4 \\
\hline Bacrim & 10.9 \\
\hline Paramilitares & 6.7 \\
\hline Centro Democrático & 5.4 \\
\hline
\end{tabular}

Tabla 9. Factor que impide la paz en Colombia. 


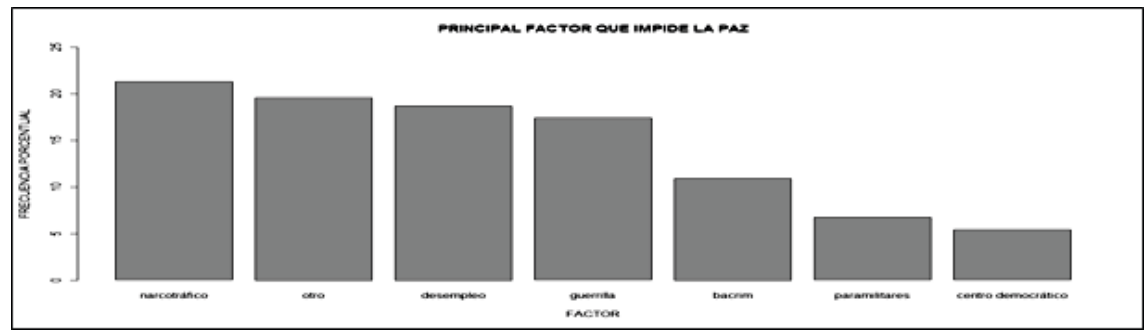

Gráfico 7. Principales factores que impiden la paz en Colombia.

Los datos muestran que el narcotráfico (21.3\%), la inseguridad (19.6\%), el desempleo (18.7\%) y la guerrilla (17.4\%), constituyen, según los EU la representación sobre los obstáculos de la paz. El tema del narcotráfico asociado a uno de los principales obstáculos a la creación de una cultura de paz en el país se encuentra en diversos estudios y publicaciones (Fernández, 2002; Gehring \& Cuervo, 2013).

El estudio también muestra, la manera como los jóvenes universitarios representan las prioridades que debe asumir el gobierno ante la firma del acuerdo de paz:

\begin{tabular}{|l|l|}
\hline \multicolumn{1}{|c|}{$\begin{array}{c}\text { Prioridad del gobierno al firmarse el } \\
\text { acuerdo de paz }\end{array}$} & \multicolumn{1}{|c|}{$\begin{array}{c}\text { Frecuencia } \\
\text { porcentual }\end{array}$} \\
\hline $\begin{array}{l}\text { Cambios en la estructura política, } \\
\text { económica y educativa }\end{array}$ & 35.0 \\
\hline Pedagogía para la paz & 24.6 \\
\hline Condenas severas a la guerrilla & 22.9 \\
\hline Trabajo a los desmovilizados & 5.3 \\
\hline Indulto a los líderes de la guerrilla & 5.3 \\
\hline Participación política de la guerrilla & 4.0 \\
\hline Otra & 2.9 \\
\hline
\end{tabular}

Tabla 10. Prioridad del Gobierno al firmarse el acuerdo de paz

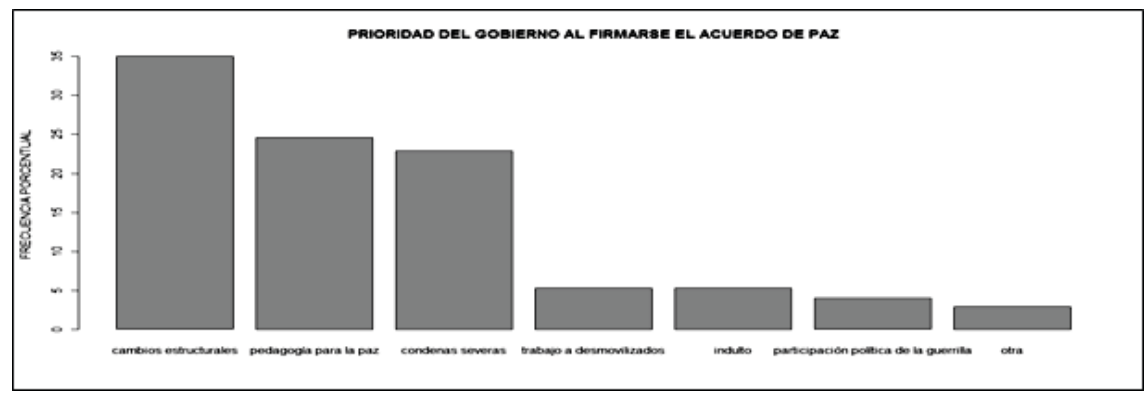

Gráfico 8. Prioridad del Gobierno si se firma el acuerdo de paz. 
La tabla No. 10 y el gráfico № 8 muestran la necesidad de implementar tres políticas estatales si se llega a un acuerdo de paz: la necesidad urgente de realizar cambios en la estructura política, económica y educativa del país (35.0\%), desarrollar una pedagogía para la paz (24.6\%) y establecer penas severas para la guerrilla (22.9\%).

\section{Representaciones Sociales sobre Paz}

Una de las categorías claves para la presente investigación consistió en reconocer la RS sobre paz. Para los EU las palabras que mejor representan la paz son la justicia (34.8\%), la equidad (16.5\%), mayores oportunidades para los jóvenes profesionales (16.5\%), y la tolerancia (12.2\%). La tabla No. 11 y el gráfico No. 9 permiten evidenciar estos datos:

\begin{tabular}{|l|l|}
\hline $\begin{array}{c}\text { Palabra que mejor representa la paz en } \\
\text { Colombia }\end{array}$ & $\begin{array}{c}\text { Frecuencia } \\
\text { porcentual }\end{array}$ \\
\hline Justicia & 34.8 \\
\hline Equidad & 16.5 \\
\hline Oportunidades & 16.5 \\
\hline Tolerancia & 12.2 \\
\hline Respeto & 11.8 \\
\hline Honestidad & 3.5 \\
\hline Otra & 4.7 \\
\hline
\end{tabular}

Tabla 11. RS sobre paz en Colombia.

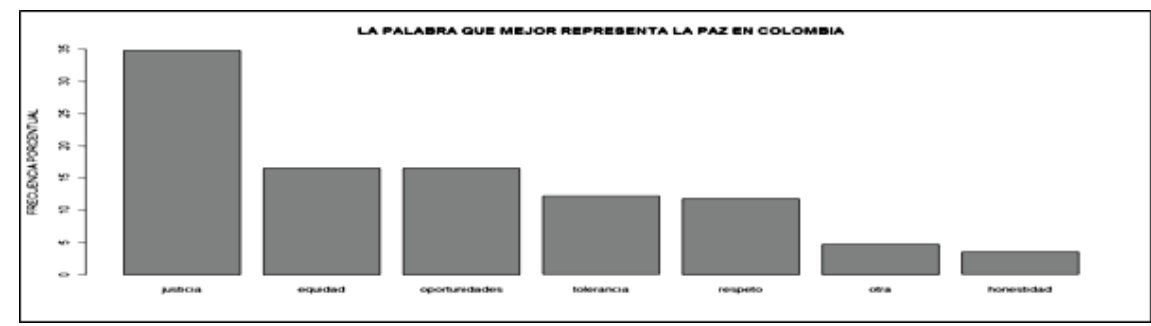

Gráfico 9. La paz para los estudiantes universitarios.

Los estudiantes universitarios representan el papel de la universidad como la institución clave que debe fortalecer el proceso de paz de manera mayoritaria en un $45.8 \%$, es decir, le asignan un papel protagónico; el $13.3 \%$ reafirma lo anterior al considerar que el rol de la universidad debe 
ser activo, y finalmente, el 11.4\% afirma que la metodología clave son los foros y seminarios. La tabla No. 12 y el gráfico No. 10 así lo exponen:

\begin{tabular}{|l|l|}
\hline \multicolumn{1}{|c|}{$\begin{array}{c}\text { Papel de la universidad en el proceso } \\
\text { de paz }\end{array}$} & \multicolumn{1}{|c|}{$\begin{array}{c}\text { Frecuencia } \\
\text { porcentual }\end{array}$} \\
\hline Fortalecer una cultura de paz & 45.8 \\
\hline Activo & 13.3 \\
\hline Difusión a través de foros y seminarios & 11.4 \\
\hline Empoderar a las comunidades & 10.6 \\
\hline Investigación académica & 7.8 \\
\hline Imparcial & 5.3 \\
\hline Proyectos de extensión & 4.7 \\
\hline Otra & 1.1 \\
\hline
\end{tabular}

Tabla 12. RS sobre paz en Colombia.

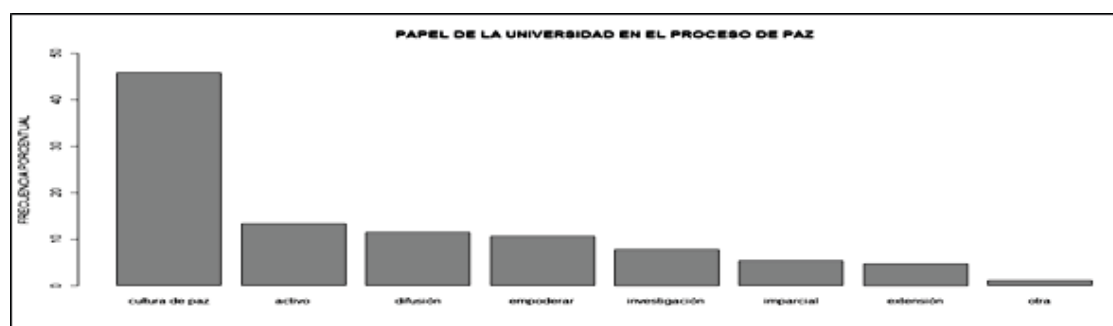

Gráfico 10. El papel de la universidad en el proceso de paz.

Otra de las preguntas orientadas a indagar en las RS sobre paz en los estudiantes universitarios, se enfocó hacia el compromiso que estos jóvenes asumirían en una eventual fase de posconflicto. A pesar del pesimismo y desesperanza sobre el éxito de los diálogos de paz, las respuestas a éste interrogante permiten ver la otra cara de la moneda: El $60.3 \%$ estaría dispuesto a difundir la cultura de paz y el $24.3 \%$ a participar activamente. Sólo el $2.9 \%$ manifiesta que no se compromete:

\begin{tabular}{|c|c|}
\hline $\begin{array}{l}\text { ¿A qué se compromete usted con la } \\
\text { paz? }\end{array}$ & $\begin{array}{l}\text { Frecuencia } \\
\text { porcentual }\end{array}$ \\
\hline Difundir la cultura de paz & 60.3 \\
\hline Participar activamente & 24.3 \\
\hline Ser imparcial & 8.7 \\
\hline No me comprometo & 2.9 \\
\hline Otra & 2.9 \\
\hline Me opongo & 0.9 \\
\hline
\end{tabular}

Tabla 13. El compromiso de los EU con la Paz. 


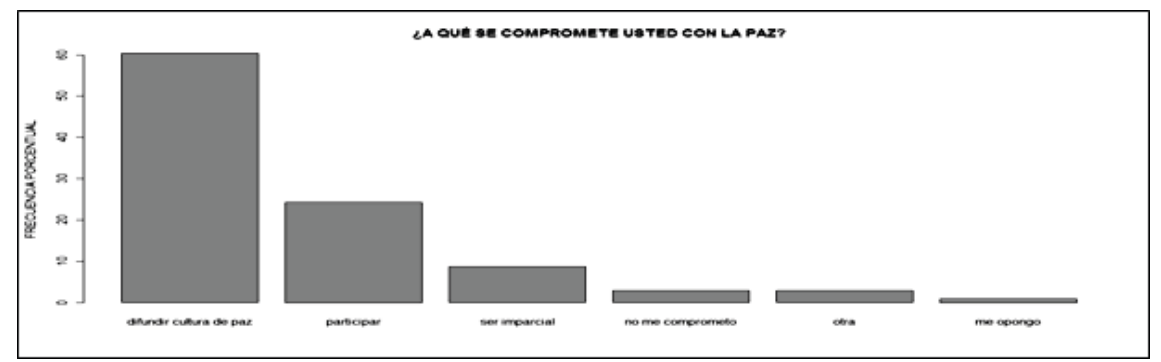

Gráfico 11. A qué se comprometen los EU ante un eventual cese del conflicto armado en el país.

\section{Representaciones sociales sobre cultura de Paz}

Para el $66.5 \%$ de los estudiantes universitarios, la cultura de paz significa "conjunto de hábitos, costumbres y saberes para resolver de forma pacífica los conflictos"; y para un $15.5 \%$ cultura de paz significa "comportamiento paciente e inteligente para controlar las emociones violentas":

\begin{tabular}{|l|l|}
\hline \multicolumn{1}{|c|}{ ¿Qué significado tiene para usted la cultura de paz? } & \multicolumn{1}{|c|}{$\begin{array}{c}\text { Frecuencia } \\
\text { porcentual }\end{array}$} \\
\hline $\begin{array}{l}\text { Conjunto de hábitos, costumbres y saberes para resolver de forma pacífica los } \\
\text { conflictos }(\mathrm{A})\end{array}$ & 66.5 \\
\hline Comportamiento paciente e inteligente para controlar las emociones violentas (B) & 15.5 \\
\hline Actitud de rechazo ante palabras y comportamientos violentos (C) & 6.4 \\
\hline Ser culto, respetar a los mayores y ser honestos (D) & 4.6 \\
\hline $\begin{array}{l}\text { Ámbito específico de las expresiones artísticas, literarias y estéticas en torno a la } \\
\text { paz (E) }\end{array}$ & 3.3 \\
\hline La cultura de paz son aquellas obras que nos han dejado personajes cultos (G) & 1.0 \\
\hline Otra (F) & 2.7 \\
\hline
\end{tabular}

Tabla 14. Representaciones sociales sobre cultura de la paz.

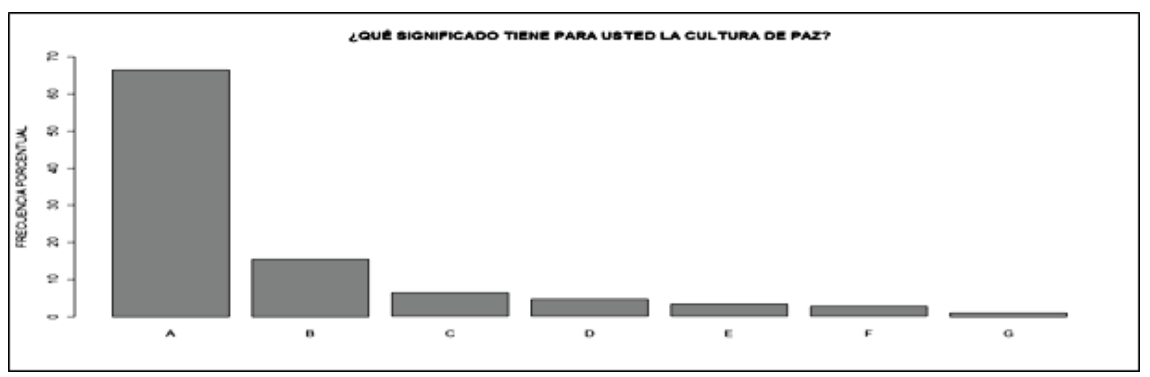

Gráfico 12. El significado de cultura de paz para estudiantes universitarios. 
La cultura de paz para los estudiantes universitarios se construye a partir de la generación de espacios de debate $(45.3 \%)$, adaptando los currículos (24.4\%), a través de la cátedra de la paz (12.7\%) y mejorando las prácticas pedagógicas (11.7\%). Llama la atención la suma de los aspectos relacionados con el currículo y las prácticas pedagógicas como estrategia para crear cultura de paz en la universidad, las cuales suman el $48.8 \%$, del total de representaciones expresados por los estudiantes:

\begin{tabular}{|l|l|}
\hline $\begin{array}{c}\text { ¿Cómo puede la universidad construir } \\
\text { cultura de paz? }\end{array}$ & \multicolumn{1}{|c|}{$\begin{array}{c}\text { Frecuencia } \\
\text { porcentual }\end{array}$} \\
\hline Generando espacios de debate & 45.3 \\
\hline Adaptando los currículos & 24.4 \\
\hline Cátedra de la paz & 12.7 \\
\hline Mejorando las prácticas pedagógicas & 11.7 \\
\hline Pedagogía del proceso de paz & 4.2 \\
\hline Otra & 1.7 \\
\hline
\end{tabular}

Tabla 15. Las estrategias de la universidad para construir cultura de paz.

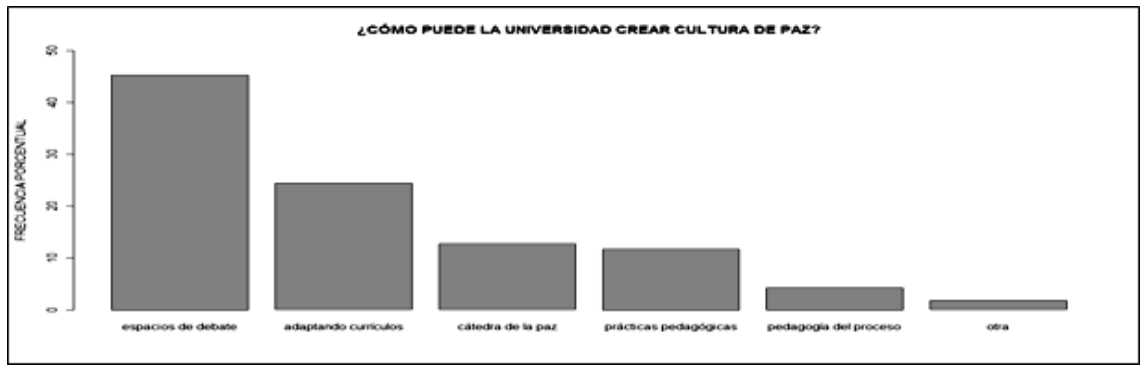

Gráfico 13. ¿Cómo puede la universidad construir cultura de paz?

La pregunta final se orientó a reconocer cuáles son las representaciones que poseen los EU sobre si existe en la universidad un ambiente favorable para construir cultura de paz. El $57.7 \%$ considera que no existe un clima propicio en la academia para llevar a cabo este singular propósito, contra un $42.3 \%$ que piensa lo contrario. La tabla No. 16 y el gráfico siguiente muestran este punto de vista:

\begin{tabular}{|l|l|}
\hline $\begin{array}{c}\text { ¿Existe un ambiente propicio } \\
\text { para construir cultura de paz en la } \\
\text { universidad? }\end{array}$ & \multicolumn{1}{c|}{$\begin{array}{c}\text { Frecuencia } \\
\text { porcentual }\end{array}$} \\
\hline Sí & 42.3 \\
\hline No & 57.7 \\
\hline
\end{tabular}

Tabla 16. Ambiente para construir cultura de paz en la universidad. 


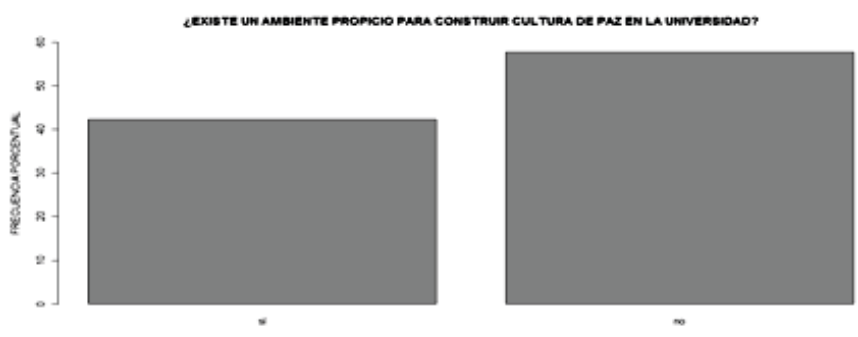

Gráfico 14. La universidad como escenario de paz.

\section{Conclusiones}

Indagar a través de una encuesta, sobre las representaciones de los jóvenes universitarios sobre cultura de paz, paz, conflicto, la universidad como escenario de paz, y otros tópicos inherentes al fenómeno estudiado, aporta una valiosa información que puede servir no sólo para la construcción de políticas institucionales y programas de formación, sino para la evaluación de los posibles factores asociados a ciertas actitudes pesimistas, y de rechazo de algún sector de la ciudadanía frente al proceso que se vive en la Habana.

Una lectura global de los resultados permite encontrar los siguientes elementos comunes:

a) Los estudiantes universitarios quieren la paz, pero siguen escépticos ante los resultados favorables que se puedan presentar en La Habana

b) Estarían dispuestos a participar, pero muestran un grado preocupante de desinformación sobre el proceso.

c) Prevalece cierta tendencia desinformativa en los jóvenes, cercana a las posiciones particulares de algún sector político del país y a la creciente ola de rumores y especulaciones que emiten ciertos medios informativos.

d) Los estudiantes universitarios asocian el logro de una cultura de paz con un cambio en las estructuras políticas, económicas y educativas del país.

e) Aunque consideran que la universidad juega un papel fundamental en la construcción de una cultura de paz, piensan 
que en las instituciones de educación superior no existe un clima favorable para lograr este propósito.

Algunos datos muestran la magnitud de estos problemas: tres (3) de cada diez (10) estudiantes manifiestan ser víctimas del conflicto armado. Del total de afectados el $46 \%$ ha sufrido la pérdida de un familiar y el $26.7 \%$ desplazamiento forzado. Del total de la muestra, el 59.9\% manifiesta desconocer los acuerdos logrados hasta la fecha en los diálogos de la Habana y el $57.7 \%$ considera que en la universidad no hay un ambiente propicio para construir una cultura de paz.

Los datos anteriores corresponden con la percepción general de muchos colombianos frente a todo este proceso: el país manifiesta cansancio ante lo que consideran "lentitud y dilatación para firmar la paz". Hay un creciente escepticismo, fruto de la abierta oposición de algún sector político y económico del país, que ve en los diálogos de la Habana una fracturación del Estado y una aparente afectación de sus intereses. La estrategia de desinformación a través de rumores, incluso de infiltración a la mesa de negociaciones, utilizando las redes sociales y los medios de comunicación, generan en la ciudadanía un pesimismo creciente y una incredulidad sobre unos posibles resultados positivos a favor del cese al conflicto armado. Aun así, entre el 2012 y el 2015 la confianza de los colombianos en el proceso se mantuvo entre el $39 \%$ y el 42\% (FIP: Fundación Ideas para la Paz).

Ahora bien, los resultados de la investigación permiten aproximarse a una interesante representación de los estudiantes universitarios sobre la cultura de paz. A pesar de la desinformación y del pesimismo, los jóvenes tienen claro que la paz no se logra con la eventual firma del cese al conflicto armado entre las guerrillas y el Gobierno colombiano. Temas como la inseguridad en las principales ciudades, la corrupción, la impunidad, la problemática social, el narcotráfico, la justicia y la necesidad de un cambio en la estructura socio-política del país, se asocian y se representan como asuntos primordiales a la hora de querer configurar una cultura de la paz no sólo en las instituciones de educación superior, sino en el país en general.

Finalmente, es importante destacar la contradicción de los estudiantes universitarios frente al proceso de paz: hay un respaldo a la salida negociada al conflicto armado, pero persiste una reticencia frente a las concesiones que el gobierno debe darle a los desmovilizados en materia 
de justicia y de participación política. El dato es contundente: a pesar de considerar que la prioridad para el gobierno al firmarse el acuerdo de paz son los cambios en la estructura política (35.0\%), el $22.9 \%$ aprueba condenas severas para la guerrilla.

Estos resultados muestran la falta de una política coherente y persistente de información a los colombianos por parte del gobierno sobre los avances del proceso de paz. Más efectivo ha resultado el clima de desinformación de ciertos sectores políticos y económicos que a través de las redes sociales y los medios, han logrado influir en la opinión pública a través de una campaña de desinformación, desprestigio y rumores. Sobre este último tópico es necesario volver sobre las preguntas iniciales que originaron el presente texto: ¿Cuál es el papel de la educación y en particular de la universidad en una eventual fase de posconflicto?

\section{Referencias bibliográficas}

Acevedo Zapata, S. (2014). Inclusión digital y educación inclusiva. Aportes para el diseño de proyectos pedagógicos con el uso de tecnologías de la comunicación. Revista De Investigaciones UNAD, 13(1), 41-57. doi:http://dx.doi.org/10.22490/25391887.1130

Amaya Balaguera, Y. (2015). Guía metodológica ágil, para el desarrollo de aplicaciones móviles "AEGIS-MD". Revista De Investigaciones UNAD, 14(1), 97 - 113.

Colombia.com (2016). Colombianos son más pesimistas ante diálogos y economía del país. Disponible en: http://www.colombia.com/actualidad/especiales/dialogos-de-paz/ noticias/sdi282/119819/colombianos-son-mas-pesimistas-ante-dialogos-y-economia-delpais

Curle, A. (1978). Conflictividad y pacificación. Barcelona: Herder.

Departamento Nacional de Estadística -DANE-. (2015). Boletín técnico sobre Convivencia y seguridad ciudadana en Cúcuta, Disponible en: https://www.dane.gov.co/files/ investigaciones/poblacion/convivencia/2015/ECSC2015-Cucuta.pdf

Fajardo Pascagaza, E. (2016). Hacia la caracterización de los valores democráticos y ciudadanos de los estudiantes universitarios: una mirada desde la formación política y la construcción de escenarios de paz. Revista Interamericana de Educación, Pedagogía y Estudios Culturales, Vol 9, Núm. 1. Disponible en: http://revistas.usta.edu.co/index.php/ riiep/article/view/3604/3499. 
Fajardo, D. (2014). Estudio sobre los orígenes del conflicto social armado, razones de su persistencia y sus efectos más profundos en la sociedad colombiana. Bogotá: Centro de Memoria Histórica. Disponible en: https://www.centrodememoriahistorica.gov.co/ descargas/comisionPaz2015/FajardoDario.pdf

Fernández, E.M. (2002). El narcotráfico y la descomposición política y social. Bogotá: Plaza y Valdés.

Fisas, V. (1998). Cultura de paz y gestión de conflictos. Barcelona: Icaria / Unesco.

Fundación Ideas para la Paz (2015). El termómetro de la paz. Disponible en: http://www. ideaspaz.org/especiales/termometro/\#p2)

Galtung, J. (1969). Violence, Peace and Peace Research, Journal of Peace Research, 6(3), 167-191.

Galtung, J. (1985). Sobre la paz. Barcelona: Fontamara.

Galtung, J. (2003a). Paz por medios pacíficos. Bilbao: Bakeaz.

Galtung, J. (2010). Futuro de la investigación para la paz. En: Todavía en búsqueda de la paz. Zaragoza: Fundación Seminario de Investigación para la Paz (SIP).

Gehring, H. \& Cuervo, M. (2013). Colombia: un largo y difícil camino hacia la paz. Disponible en: http://www.kas.de/wf/doc/kas_34649-1522-4-30.pdf?130613182757

Habermas, J. (1984). Ciencia y técnica como ideología. Tecnos: Madrid.

Jares, X. (1999). Educación para la paz, su teoría y su práctica. Madrid: Popular.

Jiménez Mendoza, W., Luciano Alipio, R., \& Soto Carrión, C. (2014). Influencia de las fuentes de financiamiento externo en el crecimiento y desarrollo de las empresas agroindustriales del distrito de Abancay Apurímac Perú (periodo 2012- 2013). Revista De Investigaciones UNAD, 13(1), 261-273. doi:http://dx.doi.org/10.22490/25391887.1141

Leclercq, J. \& Rodríguez, G. (Coords.). (2015). Índice Global de Impunidad 2015. México: Universidad de las Américas de Puebla.

Lederach, J. P. (2008). La imaginación moral: el arte y el alma de construir la paz. Bogotá: Norma.

Lederach, J.P. (2000). El abecé de la paz y los conflictos: educación para la paz. Madrid: Catarata.

Mesa, M. (2009). Paz y seguridad. En: Manual de educación para la sostenibilidad, Unescp-Etxea. Disponible en: http://www.ceipaz.org/images/contenido/16_paz_ seguridad_castellano.pdf 
Martínez Guzmán, V. (2000). Saber hacer las paces. Epistemologías de los estudios para la paz. Convergencia Revista de Ciencias Sociales, 7(23), 49-96.

Muñoz, F. (2001). La paz imperfecta. Granada: Universidad de Granada, Instituto de la Paz y los Conflictos.

Muñoz, F. (2010). ¿Cómo investigar para la paz? Una perspectiva conflictiva, compleja e imperfecta. En: Todavía en búsqueda de la paz. Zaragoza: Fundación Seminario de Investigación para la Paz (SIP).

Naidú, M.V. (1986). Dimensions of peace. Peace Research, 18(2), 3-14.

Perera, M. (2003). A propósito de las Representaciones Sociales: Apuntes teóricos trayectoria y actualidad. Disponible en: http://biblioteca.clacso.edu.ar/ar/libros/cuba/ cips/caudales05/Caudales/ARTICULOS/ArticulosPDF/02P075.pdf

Reardon, B. (1978). El desarme y la educación para la paz. Revista Perspectivas, vol. VIII(4), 437-449.

Red de ciudades cómo vamos. (2016). Seguridad ciudadana. Disponible en: http:// redcomovamos.org/servicios-publicos/

Régio, L., Egry, E., \& Apostólico, M. (2015). Consideraciones acerca de la importancia del estudio de saberes necesarios para el afrontamiento de la violencia infantil en la atención primaria de salud. Revista De Investigaciones UNAD, 14(2), 137-149. doi:http://dx.doi. org/10.22490/25391887.1463

Rojas Mesa, J., \& Leal Urueña, L. (2014). Entre flujos y fronteras: la educación superior mediada tecnológicamente vista a través de una perspectiva etnográfica. Revista De Investigaciones UNAD, 13(2), 9-27. doi:http://dx.doi.org/10.22490/25391887.1143

Sanchéz Rodríguez, C. (2016). Una propuesta epistemológica para la sistematización de experiencias generadas desde la reflexión sobre la práctica. Revista Interamericana de Educación, Pedagogía y Estudios Culturales, Vol 9, Núm. 2. Disponible en: http:// revistas.usta.edu.co/index.php/riiep/article/view/3612/3519

Semana. (2016). La mayoría de los colombianos son pesimistas sobre el proceso de paz. Semana. Disponible en: http://www.semana.com/nacion/articulo/encuesta-colombiaopina-los-colombianos-son-pesimistas-sobre-el-proceso-de-paz/464869

Transparencia internacional. (2015). Percepción de la corrupción índice 2015. Disponible en: http://www.transparency.org/cpi2015

Tuvilla, J. (2006). Cultura de paz y educación para la ciudadanía democrática. Disponible en: http://www.aecidcf.org.co/documentos/MI\%2011.669.pdf

Unesco. (1974). Resolución: contribución de la UNESCO a la Paz. París. 
Urbina, J. (2012). La pasión de aprender, el punto de vista de los estudiantes universitarios. Tesis doctoral. Manizales: CINDE- U. de Manizales.

Urbina, J. \& Muñoz, G. (2012). Ideas de Paz en jóvenes desplazados. Revista Latinoamericana de Ciencias Sociales, 9(1). Disponible en: http://revistaumanizales. cinde.org.co/index.php/Revista-Latinoamericana/article/view/359 